\title{
Hyperprolactinémie et fonction sexuelle chez l'homme
}

\author{
Jacques BUVAT, Gilbert BOU-JAOUDE
}

Centre ETPARP. Lille, France

\begin{abstract}
RESUME
La dysfonction érectile (DE), le plus souvent associée à une diminution du désir sexuel, et parfois à des dysfonctions orgasmique ou éjaculatoire, est le principal symptôme révélateur de l'hyperprolactinémie (HPRL) masculine, pathologie à ne pas méconnaître car elle est souvent associée à un adénome hypophysaire qui peut exposer à des complications sérieuses. On attribue fréquemment les dysfonctions sexuelles des hommes hyperprolactinémiques à une diminution de la sécrétion de testostérone. En fait la testostérone plasmatique est normale chez beaucoup d'entre eux. II existe d'autres mécanismes, indépendants de la testostérone, impliquant probablement des neurotransmetteurs cérébraux. Le dosage systématique de la prolactine ( $P R L)$ sérique chez les hommes consultant pour DE n'a trouvé que des prévalences faibles d'HPRL franche (>35 $\mathrm{ng} / \mathrm{ml}: 0,62 \%$ dans une compilation de 10 grandes séries) et d'adénomes hypophysaires $(0,38 \%$ parmi les même séries). L'association HPRL-DE pouvait n'être parfois qu'une coïncidence sans relation de cause à effet puisque les macroprolactines, variants biologiques inactifs ou peu actifs de la PRL, peuvent être dosées par la plupart des méthodes immunologiques courantes et être prises à tort pour de la PRL active. Leur identification nécessite une chromatographie de la PRL, qui relève de laboratoires hautement spécialisés. II n'y a pas actuellement de consensus en ce qui concerne le dépistage de I'HPRL dans la DE : un dosage systématique de la PRL pourrait se justifier puisque I'HPRL est une maladie sérieuse mais corrigeable par un traitement spécifique, alors qu'aucun critère fiable, ou au moins validé, que ce soit clinique, psychométrique ou hormonal (y compris le dosage de testostérone sérique) ne permet de limiter les dosages à certaines catégories d'hommes sans risquer de méconnaître une proportion notable des HPRL. En cas d'HPRL franche confirmée par un second dosage, la recherche d'une tumeur hypophysaire ou hypothalamique est indispensable. Les agonistes de la dopamine constituent le traitement de première intention pour les dysfonctions sexuelles résultant d'une HPRL. L'association d'une prise en charge psycho-sexologique est parfois nécessaire pour un résultat optimal.
\end{abstract}

Mots clés : hyperprolactinémie, dysfonction érectile, inhibition du désir sexuel, anorgasmie, anéjaculation, éjaculation retardée, revue

\section{INTRODUCTION}

On ne connaît pas de rôle physiologique à la prolactine (PRL) dans le contrôle du comportement sexuel humain. L'augmentation de sa sécrétion qu'induit physiologiquement l'orgasme dans les deux sexes pourrait cependant contribuer au mécanisme de la satiété sexuelle puisqu'elle n'a pas été observée chez un homme multi-orgasmique [20]. Par contre tous les types d'hyperprolactinémie (HPRL), qu'elles soient idiopathiques, ou résultent d'une prise médicamenteuse ou d'une tumeur, peuvent inhiber la plupart des aspects du comportement sexuel masculin. Bien que I'HPRL soit relativement rare chez l'homme, on doit y penser face à plusieurs dysfonctions sexuelles masculines car elle révèle souvent l'existence d'adénomes hypophysaires : micro-adénomes, définis par une taille de moins de $10 \mathrm{~mm}$, ou plus fréquemment chez l'homme macro-adénomes, de $10 \mathrm{~mm}$ ou plus. II s'agit le plus souvent de prolactinomes, constitués exclusivement de cellules lactotropes. Mais d'autres types histologiques sont possibles, l'HPRL résultant alors le plus souvent de l'interruption du contrôle dopaminergique inhibiteur exercé par l'hypothalamus du fait d'une compression de la tige hypophysaire par la tumeur. Les macro-adénomes exposent à des complications sérieuses, endocriniennes (hypopituita-

Correspondance :

Dr Jacques BUVAT - Centre ETPARP, 3 rue Carolus, 59000 Lille, France - Tel + 33320935070 -

Fax +33 320939846 - Email jacques@buvat.org 
risme, qui peut être fatal à l'occasion d'une décompensation), visuelles (hémianopsie bitemporale et au maximum cécité en cas de poussée évolutive) ou tumorales, dues à la croissance de la tumeur.

\section{PROBLÈMES SEXUELS DES HOMMES HYPERPROLACTINÉMIQUES}

Dans une revue antérieure de la littérature ayant inclus plus de 300 hommes avec HPRL, nous avons relevé des dysfonctions sexuelles dans $88 \%$ des cas, incluant chaque fois une dysfonction érectile (DE) [7]. Le profil le plus typique associait DE et diminution du désir sexuel. Quelques hommes décrivaient aussi une éjaculation retardée ou absente ou une anorgasmie, mais quasiment jamais isolément. Quelques cas d'éjaculation rétrograde ont également été rapportés, parfois curables par la bromocriptine [21]. Les autres symptômes de l'HPRL étaient inconstants : diminution de la pilosité corporelle dans $40 \%$ des cas, gynécomastie dans seulement $21 \%$ des cas, galactorrhée dans $13 \%$. La dysfonction érectile est donc le principal symptôme révélateur de l'HPRL chez l'homme.

Carani et al. [10] ont enregistré avec un Rigiscan ${ }^{\circledR}$ les érections de 6 hommes avec PRL sérique dépassant 300 $\mathrm{ng} / \mathrm{ml}$ et présentant donc a priori un prolactinome : leurs érections nocturnes, comme celles induites par stimulation sexuelle audiovisuelle, ne différaient pas de celles d'hommes témoins normaux. Par contre De Rosa et al. [13], pratiquant la même exploration chez 51 autres hommes HPRL pendant 3 nuits consécutives, et utilisant comme critère de normalité la présence au cours d'une nuit de 3 épisodes de rigidité d'au moins $10 \mathrm{mn}$ et $>70 \%$ sur l'échelle du Rigis$\operatorname{can}^{\circledR}$, ont conclu à la présence d'anomalies chez $100 \%$ (41/41) de leurs patients avec macroprolactinome et $80 \%$ $(8 / 10)$ de ceux avec microprolactinome, mais aucun des 44 témoins normaux de moins de 50 ans. La proportion des hommes avec anomalies des érections nocturnes $(96,7 \%)$ était très largement supérieure à celle des hommes qui avaient remarqué une altération de leurs érections $(27 / 51$, $53 \%$ ).

\section{MÉCANISMES DES PROBLÈMES SEXUELS DES HOMMES HYPERPROLACTINÉMIQUES}

L'HPRL inhibe la sécrétion pulsatile de $\mathrm{LH}$, et diminue ainsi celle de la testostérone. On croit souvent que c'est cet hypogonadisme qui est la principale cause de la DE. En fait de nombreuses constatations prouvent qu'il ne peut expliquer tous les cas. Quoique généralement basse, la testostérone reste en effet dans la zone normale chez près de la moitié des hommes avec HPRL marquée et DE (par exemple 7 fois sur 16 dans une série personnelle [7], comme chez 11 des 41 macroadénomes et 5 des 10 microadénomes de De Rosa et al. [13]). De plus, la Sex Hormone Binding Globulin est abaissée chez les hommes HPRL [40], ce qui atténue les effets de la baisse de la testostérone totale en augmentant la proportion de sa fraction non liée, dite libre, principale fraction biologiquement active.

Egalement, parmi 51 hommes HPRL, De Rosa et al. [13] ont trouvé une corrélation inverse hautement significative $(p=0,0002)$ entre érections nocturnes et PRL, mais pas testostérone ni âge. II faut noter qu'ils ont aussi trouvé une corrélation négative, quoique moins significative, entre érections nocturnes et $P R L(p<0,02)$, ainsi que cette fois une corrélation positive entre érections nocturnes et testostérone, chez leurs 51 témoins. De même, au cours du traitement des hommes HPRL par l'agoniste dopaminergique bromocriptine, l'amélioration de la fonction sexuelle et celle des érections nocturnes sont mieux corrélées à la diminution de la PRL qu'à l'augmentation de la testostérone [7, 13]. Particulièrement les érections sexuelles ou nocturnes peuvent réapparaître avant toute augmentation de la testostérone si la PRL a suffisamment diminué. D'autres études, y compris celle de Bancroft [3] qui a comparé en double-insu bromocriptine et placebo chez un seul patient, tendent à supporter l'hypothèse d'un impact direct de l'HPRL sur le comportement sexuel de l'homme, indépendant de son impact sur la testostérone.

Ces mécanismes indépendants de la testostérone peuvent inclure la diminution de sa 5 alpha réduction en dihydrotestostérone rapportés par Lobo et Kletzky [27] chez les hommes HPRL, puisque la dihydrotestostérone semble le principal métabolite responsable des effets de la testostérone sur le cerveau des primates. Mais les plus importants se situent probablement au niveau d'interactions entre taux élevés de PRL et systèmes de neurotransmetteurs. La PRL augmente la synthèse, le turn-over et la libération de la dopamine à partir des neurones hypothalamiques, ce qui pourrait expliquer l'effet biphasique de l'HPRL chez le rat [15]. La dopamine stimule en effet le comportement sexuel dans la plupart des espèces animales. L'augmentation de sa libération pourrait donc expliquer l'effet stimulant initial de l'HPRL sur la fonction sexuelle du rat. Dans un second temps une désensibilisation des récepteurs à la dopamine, conséquence de leur stimulation excessive, expliquerait son effet inhibiteur secondaire. De plus l'HPRL induit une augmentation franche de l'expression de l'ARN messager de la tyroxine hydroxylase dans les noyaux arqué et périventriculaire de l'hypothalamus, une région cérébrale impliquée dans le contrôle des fonctions sexuelles et particulièrement érectile [37]. La PRL interagit également avec les systèmes opioïdes et sérotoninergiques, tous deux impliqués dans l'ajustement du comportement sexuel [5]. Des expérimentations récentes de Rehman et al. [34] ont confirmé l'effet principalement central et indépendant de la testostérone de l'HPRL chez le rat, puisque dans cette espèce les réflexes péniens sont abolis par des injections de PRL alors que les mécanismes périphériques de l'érection restent intacts, et que ces réflexes péniens ne sont pas rétablis par la supplémentation en testostérone.

\section{PRÉVALENCE DE L'HYPERPROLACTINÉMIE CHEZ LES HOMMES CONSULTANT POUR DYSFONCTION ÉRECTILE}

Le dosage systématique de la PRL chez les hommes consultant pour DE n'a trouvé que des prévalences très faibles d'HPRL ( 1 à $5 \%$ ). La compilation des 10 séries les plus importantes de la littérature (Tableau 1) conclut à des 
Tableau 1 : Prévalence de l'hyperprolactinémie et des tumeurs hypophysaires chez les hommes consultant pour Dysfonction Erectile (10 séries totalisant 8707 cas).

\begin{tabular}{|c|c|c|c|c|c|}
\hline Auteurs & Année & $\begin{array}{l}\mathrm{Nb} \\
\text { cas }\end{array}$ & $\begin{array}{c}\text { PRL > } \\
18-25 \mathrm{ng} / \mathrm{ml} \\
\end{array}$ & $\begin{array}{c}\text { PRL > } \\
35-50 \mathrm{ng} / \mathrm{ml}\end{array}$ & $\begin{array}{c}\text { Tumeur } \\
\text { hypophysaire }\end{array}$ \\
\hline \multicolumn{6}{|l|}{ Maatman et } \\
\hline Montague [28] & 1986 & 300 & & 4 & 1 \\
\hline Leonard et al. [26] & 1989 & 1236 & & $?$ & 4 \\
\hline Kropman et al. [24] & 1991 & 377 & & 2 & 1 \\
\hline Johnson et Jarow [22] & 1992 & 330 & & 1 & 1 \\
\hline Apkunonu et al. [1] & 1994 & 299 & & 3 & 0 \\
\hline Buvat et Lemaire [6] & 1997 & 1821 & 22 & 12 & 7 \\
\hline Delavierre et al. [14] & 1999 & 445 & $9 \mathbf{a}$ & 4 & 0 \\
\hline Johri et al. [23] & 2001 & 138 & & 3 & 4 \\
\hline Earle et Stuckey [16] & 2003 & 1455 & 18 & $7 \mathbf{b}$ & 6 \\
\hline Bodie et al. [4] & 2003 & $2306 c$ & & $10 d$ & $9 \mathbf{e}$ \\
\hline Total & & 8707 & $\begin{array}{c}49 / 3721 \\
(1,3 \%)\end{array}$ & $\begin{array}{l}46 / 7441 \\
(0,62 \%)\end{array}$ & $\begin{array}{c}33 \\
(0,38 \%)\end{array}$ \\
\hline
\end{tabular}

a : 8 HPRL iatrogènes;

b : 1 cas iatrogène, DE améliorée par la bromocriptine dans les 6 cas avec tumeur hypophysaire ;

c : tous âgés de plus de 50 ans, pas d'information sur un éventuel second dosage ;

d : tous $>50 \mathrm{ng} / \mathrm{ml}$;

e : $>50 \mathrm{ng} / \mathrm{ml}$ chez 5 patients $/ 9$, entre 18 et $50 \mathrm{ng} / \mathrm{ml}$ chez les 4 autres.

prévalences d'HPRL franches (PRL>35 $\mathrm{ng} / \mathrm{ml}$ ) de seulement $0,62 \%$ et d'adénomes hypophysaires de $0,38 \%$. Bien que faibles ces prévalences sont supérieures à celles trouvées dans des populations générales asiatiques par Miyai et al. (respectivement 0,17 et $0,036 \%$ chez 8450 hommes [29]) et Miyake et al. (respectivement 0,29 et $0,06 \% \mathrm{chez}$ 4803 hommes [30]).

De plus il a été trouvé une HPRL modérée (PRL entre 20 et $35 \mathrm{ng} / \mathrm{ml}$ ) chez certains hommes avec DE. La prévalence en était de $1,5 \%$ dans une série personnelle de 1370 cas consécutifs [8]. II est peu probable que des HPRL aussi modestes aient été la véritable cause de la DE puisque aucun des 21 cas de cette série n'avait de tumeur hypophysaire (2 micro-adénomes ont toutefois été trouvés dans des cas analogues par Johri et al. [23]) ni de diminution du taux de testostérone, tandis que l'administration de bromocriptine, un agent hypoprolactinémiant, n'a amélioré la fonction érectile que dans $40 \%$ des cas, un taux identique à celui qui a été rapporté précédemment chez des hommes avec DE et PRL normale, également traités par la bromocriptine par Ambrosi et al. [2].

Il faut toutefois aussi citer l'expérience de Caruso et al. [13] consacrée à 15 hommes avec DE modérée chez qui ils n'avaient pas trouvé d'autre anomalie franche qu'une HPRL modérée objectivée uniquement par des dosages de PRL faits à $17 \mathrm{~h}$ (moyenne $31,2+3,9 \mathrm{ng} / \mathrm{ml}$, contre seulement $8,8 \pm 1$ et $9,3 \pm 4,2 \mathrm{ng} / \mathrm{ml}$ à $8 \mathrm{~h}$ et $13 \mathrm{~h}$ ). Ces hommes n'avaient pas été améliorés par la prise sublinguale à la demande d'apomorphine, un agoniste dopaminergique faible. Après administration quotidienne du même médicament pendant 1 mois, ils ont observé une normalisation de la PRL dosée à $17 \mathrm{~h}$ (moyenne 10,6 +5,3 $\mathrm{ng} / \mathrm{ml}$ ) et une amélioration de la satisfaction sexuelle chez 13 patients sur 15, corroborée par des augmentations significatives des scores des domaines Fonction Erectile, Désir Sexuel, Satisfaction des rapports et Satisfaction d'ensemble de l'Index International de la Fonction Erectile (IIEF).

A cette exception près, qui reste à confirmer, l'effet des traitements hypoprolactinémiants n'est convaincant qu'en cas d'HPRL franche. Ainsi la bromocriptine a amélioré à elle seule de façon significative la fonction érectile de 8 de nos 12 cas de DE avec PRL > $35 \mathrm{ng} / \mathrm{ml}$ (67\%) [6]. Les 4 autres patients restaient incapables de pénétrer leur partenaire malgré la normalisation de leur PRL mais décrivaient une amélioration de leur libido et de leurs érections matinales, et une prise en charge psycho-sexologique complémentaire indiquée par l'association de facteurs psychologiques patents finit d'en guérir deux avec retour d'érections coïtales normales.

Le groupe de Masters et Johnson a aussi rapporté dans une étude non contrôlée que la fonction sexuelle des hommes avec HPRL franche était mieux améliorée par la bromocriptine que par psycho ou sexothérapie [36]. 


\section{MACROPROLACTINÉMIES OU FAUSSES HYPERPROLACTINÉMIES}

Avant d'attribuer la responsabilité d'une dysfonction sexuelle à une HPRL, il faut se souvenir que des variants biologiques inactifs ou peu actifs de la PRL peuvent être dosés par les méthodes immunologiques courantes, et simuler une HPRL s'ils sont secrétés en excès. II s'agit particulièrement des macroprolactines (macroPRL), «big» et «big-big prolactin», qui ont des poids moléculaires de respectivement 50-60 et 150 kilo Daltons, au lieu des 22 kilo Daltons de la PRL biologiquement active [38]. Un excès de ces macroPRL n'a généralement pas de conséquence pathologique. II rend compte de $10 \%$ des HPRL [39], et on l'observe habituellement chez des individus par ailleurs normaux, principalement des femmes avec fonction reproductrice préservée en dépit de l'HPRL. On peut aussi en trouver, par simple coïncidence, chez les hommes avec DE $[25,19]$. En tel cas, le taux de testostérone plasmatique est habituellement normal, comme le sont le scanner et l'examen en Résonnance Magnétique Nucléaire (IRM) de la région hypothalamo-hypophysaire. Les agents hypoprolactinémiants comme la bromocriptine normalisent les taux de (macro) PRL mais sont incapables d'améliorer la fonction sexuelle. Tout ceci doit faire évoquer le fait que l'HPRL ne soit pas la cause de la DE. II faut alors faire réaliser une analyse chromatographique de la PRL dans un laboratoire spécialisé en endocrinologie, ce qui permet l'identification de la macroPRL et redresse le diagnostic. II a été recommandé que tous les fabricants de dosages de PRL évaluent la spécificité de leur propre dosage par rapport aux macroPRL et en informent les utilisateurs, et par ailleurs que les laboratoires dosant la PRL pratiquent une détection systématique des macroPRL, mais ces recommandations n'ont pas pour le moment été suivies d'effet [17].

Les HPRL masculines répertoriées dans les séries les plus anciennes du Tableau 1 ont certainement inclus quelques macroprolactinémies non identifiées. Le fait qu'en dosant systématiquement pendant plusieurs décades la PRL chez nos patients avec DE nous ayons nous-même observé une tendance à la diminution de la prévalence des HPRL avec les années (Tableau 2) [6] pourrait s'expliquer par l'amélioration progressive de la spécificité des dosages aboutissant à moins de confusion avec les macroprolactinémies. Une autre explication pourrait être le nombre probablement important d'HPRL anciennes qui attendaient d'être diagnostiquées il y a 25 ans, au moment de la mise au point du dosage de la PRL. Elles ont pu augmenter artificiellement la prévalence des HPRL au cours des premières années d'utilisation de ces dosages.

Un diagnostic de macroprolactinémie ne doit cependant faire exclure ni l'Imagerie en Résonnance Magnétique (IRM), puisque quelques cas ont été trouvés associés à des adénomes hypophysaires [39, 31], ni un essai de traitement par agent hypoprolactinémiant, puisqu'on a observé une certaine activité biologique de la macroPRL jusque chez un tiers des femmes avec macroprolactinémie, avec dans quelques cas correction de l'aménorrhée et de l'infertilité en résultant sous agoniste de la dopamine [39].

\section{PRÉVALENCE DE L'HPRL DANS LES AUTRES DYSFONCTIONS SEXUELLES MASCULINES}

Nous avons dosé systématiquement la PRL chez des hommes vus consécutivement pour inhibition du désir sexuel sans $D E(n=53)$, éjaculation retardée, anéjaculation ou anorgasmie $(n=74)$ et éjaculation prématurée $(n=124)[7$, 8]. Nous n'avons trouvé aucun cas d'HPRL dans les deux premières catégories. Cependant nos séries étaient courtes, et Schwartz et al. [36] ont observé quelques cas d'HPRL chez des hommes consultant pour désir sexuel hypoactif isolé, ou anorgasmie. Par contre nous avons trouvé des taux de PRL modérément élevés (20 à 35 $\mathrm{ng} / \mathrm{ml}$ ) chez 13 des 124 hommes avec éjaculation prématurée $(10 \%)$. Cette HPRL n'était pas a priori la cause de la dysfonction sexuelle puisque dans aucun des cas le traitement par la bromocriptine n'allongea la latence éjaculatoire. De pius la testostérone plasmatique était normale dans tous les cas et il ne fut trouvé aucun adénome hypophysaire chez ces hommes.

Tableau 2 : Prolactinémie basale chez 1821 sujets ayant consulté notre centre pour Dysfonction Erectile pendant 20 ans. Le dosage fut systématique jusqu'en 1988, puis limité aux sujets avec manque d'intérêt sexuel, gynécomastie ou galactorrhée, ou testostérone plasmatique $<4 \mathrm{ng} / \mathrm{ml}$.

\begin{tabular}{|c|c|c|c|c|c|}
\hline Publication & Années & $\begin{array}{c}\text { Nombre } \\
\text { de cas }\end{array}$ & $\begin{array}{c}20 \text { à } 35 \\
\mathrm{ng} / \mathrm{ml}\end{array}$ & $\begin{array}{l}>35 \\
\mathrm{ng} / \mathrm{ml}\end{array}$ & $\begin{array}{c}\text { Tumeur } \\
\text { hypophysaire }\end{array}$ \\
\hline Buvat et al. 1982 [7] & $1976-81$ & 539 & $\begin{array}{c}12 \\
2,2 \%\end{array}$ & $\begin{array}{c}5 \\
1 \%\end{array}$ & $\begin{array}{c}4 \\
0,76 \%\end{array}$ \\
\hline Buvat et al. 1989 [8] & $1982-88$ & 831 & $\begin{array}{c}9 \\
1,1 \%\end{array}$ & $\begin{array}{c}5 \\
0,6 \%\end{array}$ & $\begin{array}{c}2 \\
0,24 \%\end{array}$ \\
\hline Buvat et Lemaire 1997 [6] & $1989-95$ & $451 a$ & $\begin{array}{c}1 \\
0,2 \%\end{array}$ & $\begin{array}{c}2 \\
0,4 \%\end{array}$ & $\begin{array}{c}1 \\
0,22 \%\end{array}$ \\
\hline Tous les cas & $1976-95$ & 1821 & $\begin{array}{c}22 \\
1,2 \%\end{array}$ & $\begin{array}{c}12 \\
0,66 \%\end{array}$ & $\begin{array}{c}7 \\
0,38 \%\end{array}$ \\
\hline
\end{tabular}

a : sur environ 1000 Dysfonctions Erectiles explorées durant cette période. 


\section{DIAGNOSTIC DES HYPERPROLACTINÉMIES CHEZ LES HOMMES AVEC DYSFONCTION SEXUELLE}

II est important de rappeler les précautions à prendre pour éviter les fausses HPRL liées au stress (spécialement lié à la prise de sang), au repas, ou à la prise de médicaments. Le prélèvement sanguin doit être fait à jeun, après $20 \mathrm{mn}$ de repos dans un endroit calme. Toute augmentation de la PRL sérique doit être vérifiée par un second dosage, si possible après avoir posé un cathéter dans la veine $20 \mathrm{mn}$ avant le prélèvement sanguin, et après arrêt de toute médication susceptible d'augmenter la PRL (Tableau 3). En cas de discordance entre taux de PRL élevés et profil clinique plutôt évocateur de dysfonction sexuelle d'origine psychologique, ou en cas d'échec d'un traitement par agoniste de la dopamine, l'utilité d'une chromatographie de la PRL devrait être discutée.

Tableau 3 : Médicaments susceptibles d'augmenter la prolactine sérique (le plus souvent du fait de leurs activités antidopaminergiques).

- Opiacés, Méthadone

- Médicaments psychotropes :

- Neuroleptiques :

- Benzamides : Amisulpride, Sulpiride, Sultopride, Tiapride - Phenotiazines : Chlorpromazine, Cyamemazine, Fluphénazine, Levomépromazine, Perphenazine, Pipetiazine, Propériciazine, Thioridazine

- Butyrophénones : Dropéridol, Halopéridol, Pipamperon

- Flupentixol, Loxapine, Olanzapine, Pimozide, Rispéridone, Zuclopenthixol

\section{- Antidépresseurs tricycliques :}

- Amitriptyline, Amoxapine, Chlorimipramine, Désipramine, Dosulépine, Doxépine, Imipramine, Maprotiline, Trimipramine

- Anti-émétiques : Métroclopramide, Métopimazide

- Anti-ulcéreux : Cimétidine (uniquement à dose élevée)

- Anti-hypertenseurs : Reserpiniques, a-méthyl-DOPA

- Estrogènes

La plupart des auteurs juge que la très faible prévalence des HPRL significatives ne justifie pas un dosage systématique de la PRL chez les hommes avec DE si l'on tient compte de la grande prévalence de cette dernière et du coût du dosage [22, 24, 1, 6, 14]. On recommande souvent de ne la doser qu'en cas de testostérone plasmatique basse, ou de désir sexuel faible.

Cependant la testostérone n'est pas abaissée chez tous les hommes HPRL. Son taux n'était inférieur à la normale $(<3 \mathrm{ng} / \mathrm{ml})$ que dans 10 des 17 cas d'une série personnelle, y compris chez seulement 5 des 10 cas consultant pour
DE [7]. II était dans la zone normale basse ( 3 à $4 \mathrm{ng} / \mathrm{ml}$ ) chez 4 des 7 autres, et supérieur à $4 \mathrm{ng} / \mathrm{ml}$ chez seulement 3. De nombreux autres cas de testostérone normale en dépit d'une HPRL marquée ont été rapportés, parmi lesquels certains avaient une tumeur hypophysaire et correspondaient donc peu probablement à des macroprolactinémies (voir revue in [7] et Tableau 1). Ne déterminer la prolactine plasmatique qu'en cas de testostérone basse aurait fait méconnaître $50 \%$ des 12 HPRL franches et 3 des 7 adénomes hypophysaires détectés dans une autre série personnelle antérieure [6].

Même le désir sexuel peut être normal chez les hommes avec DE et HPRL, ou tout au moins peut sembler normal au patient. Johri et al. n'ont pas trouvé de corrélation entre taux de PRL et désir sexuel évalué par l'interrogatoire, ou par le score du domaine désir sexuel de l'International Index of Erectile Function (IIEF) [23]. En ne déterminant la PRL sérique qu'en cas de score du domaine désir sexuel inférieur à 3 , ils auraient méconnu $50 \%$ des HPRL identifiées par le dosage systématique chez leurs patients avec DE.

Les mêmes auteurs [23] ont suggéré que l'IlEF pourrait cependant aider au screening de I'HPRL, puisqu'ils ont observé que parmi 136 hommes avec DE, les 9 cas d'HPRL sévère ou modérée avaient tous une $D E$ sévère selon les critères de l'llEF (score du domaine fonction érectile inférieur à 10). Ceci reste cependant à confirmer, puisque Carani et al. [9] n'ont au contraire trouvé que des anomalies modérées des érections chez leurs hommes HPRL (érections nocturnes normales, et réponses érectiles normales à la stimulation sexuelle audiovisuelle). En ce qui nous concerne [8], ayant dosé systématiquement la PRL sérique chez 1370 hommes avec DE, nous avons trouvé qu'en limitant le dosage à ceux qui avaient soit un désir sexuel faible, soit une gynécomastie, soit une testostérone plasmatique à moins de $4 \mathrm{ng} / \mathrm{ml}$, c'est à dire basse, ou normale basse, nous aurions évité plus de la moitié des dosages, et n'aurions manqué qu'une seule des $10 \mathrm{HPRL}$ franches découvertes dans cette série, et aucune des 6 tumeurs hypophysaires.

Le coût du dosage systématique de PRL chez les hommes consultant pour DE a été calculé par Johri et al. [23]. Ayant trouvé 3 HPRL franches parmi 138 patients, il était de 60 dollars US par cas détecté dans leur institution (coût du dosage 1,32€). II aurait été de 851 \$ par cas détecté en cas de recours à un laboratoire privé de leur région (coût du dosage $18,5 €$ ). Ils en ont conclu que le dosage systématique de la PRL était un moyen relativement peu coûteux de détecter une maladie sérieuse et corrigeable.

II n'y a donc pas actuellement de consensus en ce qui concerne le dosage de la PRL sérique chez les hommes avec $D E$, inhibition du désir sexuel et éjaculation ou orgasme retardé ou absent : doit-il être systématique, ou limité à des hommes sélectionnés selon des critères cliniques (désir sexuel faible), psychométriques (IIEF ? intérêt certainement d'abord à confirmer) et/ou hormonaux (taux de testostérone faible ou limite, $<4 \mathrm{ng} / \mathrm{ml}$ ) ? Ceci dépend avant 
tout des ressources locales. Si le dosage doit être limité à certains cas, la stratégie la plus rentable devrait se baser sur les résultats d'études complémentaires avec dosage systématique dans un plus grand nombre de cas.

Chacun s'accorde par contre sur le fait que tout homme présentant une HPRL non iatrogène, confirmée par un second dosage à partir d'un sang prélevé dans des conditions optimales, doit bénéficier d'explorations morphologiques de la région hypothalamo-hypophysaire incluant si possible une IRM afin de détecter une éventuelle tumeur. Cette exploration est particulièrement indispensable en cas de $\mathrm{PRL}>35$ $\mathrm{ng} / \mathrm{ml}$. A titre d'illustration tirée des études les plus récentes, parmi 5 hommes avec HPRL et hypogonadisme, Rhoden et al. [35] ont trouvé grâce à l'IRM 3 adénomes hypophysaires, correspondant aux 3 hommes avec PRL supérieure à 2 fois la limite supérieure de la norme $(34 \mathrm{ng} / \mathrm{ml}$ dans leur laboratoire) mais aucun en dessous de ce taux et Bodie et al. [4] ont trouvé un adénome dans $57 \%$ de leurs HPRL > 50 $\mathrm{ng} / \mathrm{ml}$. De plus en cas de macroadénome qui constitue le type tumoral le plus fréquent chez l'homme (41 cas pour 10 microadénomes dans la série de De Rosa et al. [13]), un bilan complet des fonctions hormonales hypophysaires doit être réalisé de préférence par un endocrinologue, eu égard au risque d'hypopituitarisme consécutif à la compression de la tige hypothalamo-hypophysaire, ainsi qu'à la destruction du tissu hypophysaire par la tumeur. A titre d'exemple, récemment De Rosa et al. [13] ont rapporté 10 hypothyroïdies et 3 hypocorticismes d'origine centrale parmi leurs 41 macroprolactinomes.

\section{TRAITEMENT DES HOMMES AVEC DYSFONCTION SEXUELLE ET HYPERPROLACTINÉMIE}

On trouve dans la littérature quelques améliorations franches de dysfonctions sexuelles associées à une HPRL après traitements non spécifiques tels que psychothérapie ou sexothérapie $[3,36]$ (dans la seconde étude, un traitement ultérieur par bromocriptine avait cependant obtenu des résultats supérieurs), ou en ce qui concerne la DE ou le Sildénafil $[23,18]$. Cependant les agonistes de la dopamine (bromocriptine-Bromokin ${ }^{\circledR}$ Parlodel ${ }^{\circledR}$, lisurideArolac $^{\circledR}$ Dopergine ${ }^{\circledR}$, quinagolide-Norprolac ${ }^{\circledR}$, et cabergolide-Dostinex", le dernier ayant l'avantage d'être souvent efficace en une seule prise par semaine, et de normaliser plus rapidement la PRL [12]), sont le traitement de référence et normalisent le plus souvent à eux seuls tous les aspects de la fonction sexuelle tout en diminuant la taille de l'éventuel adénome hypophysaire, ou au moins en empêchant sa croissance $[7,5,6]$. Particulièrement, ils améliorent le désir sexuel, et en cas de DE, les érections spontanées, évitant donc d'avoir à programmer le rapport sexuel comme c'est le cas avec le Sildénafil. Ce traitement pharmacologique n'est pas obligatoirement définitif. En cas de normalisation de la PRL, s'il n'existe pas de macroadénome il convient de l'interrompre périodiquement (tous les 1 à 2 ans), et de redoser la PRL après arrêt. Dans 20\% des cas environ L'HPRL ne récidive plus après quelques années [32].
En cas de tumeur hypophysaire et particulièrement de macro-adénome (> $10 \mathrm{~mm}$ de diamètre), il est souhaitable de confier d'abord le patient à un endocrinologue pour exploration complète de ses fonctions hypophysaires, et discussion de l'éventuelle indication d'une ablation chirurgicale de l'adénome. En cas de macro-adénome, celle-ci mettrait à l'abri des complications tumorales, particulièrement ophtalmologiques et évolution vers un hypopituitarisme. En cas de micro-adénome $(<10 \mathrm{~mm}$ ), la chirurgie, cette fois transphénoïdale et moins délabrante, offre une possibilité de guérison définitive de la tumeur et de l'HPRL qui l'accompagne ([12], jusque 7 fois sur 11 avec un recul moyen de 7 ans dans la série de Wolfsberger et al. [41]). On commence cependant souvent par le traitement médical même en cas de tumeur hypophysaire [12], mais la surveillance radiologique et endocrinologique est alors très importante. II faut également savoir qu'en cas de macroadénome, un hypogonadisme peut persister malgré la normalisation de la PRL par le traitement médical (du fait de la compression hypophysaire résultant d'un macroprolactinome resté volumineux) ou chirurgical (du fait d'un délabrement séquellaire de l'hypophyse ou des connections hypothalamo-hypophysaires). Dans ce cas, la persistance d'un taux faible de testostérone compromet les chances d'amélioration sexuelle et un traitement androgénique complémentaire doit être envisagé. II faut cependant savoir que l'administration de testostérone peut stimuler la croissance de l'éventuelle tumeur hypophysaire par l'intermédiaire de son aromatisation en estradiol si l'HPRL n'est pas parfaitement contrôlée par agoniste de la dopamine, et même plus exceptionnellement si elle l'est [33]. Enfin l'association d'un conseil sexuel, ou d'une sexothérapie est souvent bénéfique, et s'avère même indispensable dans certains cas, particulièrement en cas de dysfonction sexuelle primaire (c'est à dire ayant existé dès le début de la vie sexuelle).

Remerciements à Mme Joëlle Bastin pour sa préparation experte du manuscrit.

\section{REFERENCES}

1. AKPUNONU B.E., MUTGI A.B., FEDERMAN D.J., YORK J., WOLDENBERG I.S. : Routine prolactin measurement is not necessary in the initial evaluation of male impotence. J. Gen. Intern. Med., 1994, $9:$ 336-341.

2. AMBROSI B., BARA R., TRAVAGLINI P. et al. : Studies of the effects of bromocriptine on sexual impotence. Clin. Endocrinol., (Oxf.), 1987, $7: 417-420$.

3. BANCROFT J., O'CAROLL R., NEILLY A., SHAW R.W. : The effects of bromocriptine on the sexual behavior of hyperprolactinemic man : a controlled case-study. Clin. Endocrinol., 1984, 21: 131-137.

4. BODIE J., LEWIS J., SCHOW D., MONGA M. : Laboratory evaluations of erectile dysfunction : An evidence based approach. J. Urol., 2003, $169:$ 2262-2264.

5. BUVAT J. : Hormones et comportement sexuel de l'homme : données physiologiques et physiopathologiques. Contr. Fertil. Sex., 1996, $24:$ 767-778.

6. BUVAT J., LEMAIRE A. : Endocrine screening in 1022 men with erectile dysfunction : clinical significance and cost-effecti- 
ve strategy. J. Urol., 1997, $158:$ 1764-1767.

7. BUVAT J., LEMAIRE A., BUVAT-HERBAUT M., FOURLINNIE J.C., RACADOT A., FOSSATI P. : Hyperprolactinemia and sexual function in men. Hormone Res., 1985, 22 : 196-203.

8. BUVAT J., LEMAIRE A., BUVAT-HERBAUT M., MARCOLIN G. : Dosage de la prolactine chez les impuissants. Presse Med., 1989, $18: 1167$.

9. CARANI C., GRANATA A.R., FUSTINI M.F., MARRAMA P. : Prolactin and testosterone: their role in male sexual function. Int. J. Androl., 1996, $19: 48-54$.

10. CARANI C., ZINI D., BALDINI A., DELLA CASA L., GHIZZANI A., MARRAMA P. : Testosterone and prolactin : behavioural and psychophysiological approaches in men. In : Bancroft $\mathrm{J}$. ed. The pharmacology of sexual function and dysfunction. Esteve Foundation Symposia, Vol. 6. Excerpta Medica, Amsterdam, Elsevier Science, 1995 : 145-150.

11. CARUSO S., INTELISANO G., FARINA M., DI MARI L., AGNELLO C., GIAMMUSSO B. : Efficacy and safety of daily intake of apomorphine SL in men affected by erectile dysfunction and mild hyperprolactinemia : A prospective, open-label, pilot study. Urol., 2003, 62 : 922-927.

12 DE ROSA M., ZARRILLI S., DI SARNO A., et al. : Hyperprolactinemia in men. Endocrine, 2003, $20: 75-82$.

13. DE ROSA M., ZARRILLI S., VITALE G. et al. : Six months of treatment with Cabergoline restores sexual potency in hyperprolactinemic males: An open longitudinal study monitoring nocturnal penile tumescence. J. Clin. Endocr. Metab., 2004, $89: 621-625$.

14. DELAVIERRE D., GIRARD P., PENEAU M., IBRAHIM H. : Faut-il doser la prolactinémie dans le bilan d'une insuffisance érectile ? A propos d'une série de 445 patients. Revue de la littérature. Prog. Urol., 1999, 9 : 1097-1101.

15. DRAGO F., PELLEGRINI-QUARANTOTTI B., SCAPAGNINI U., GESSA G.L. : Short-term endogenous hyperprolactinemia and sexual behavior of male rats. Physiol. Behav., 1981, 26 : 277-279.

16. EARLE C.M., STUCKEY B.G.A. : Biochemical screening in the assessment of erectile dysfunction : What tests decide future therapy ? Urol., 2003, $62: 727-731$.

17. FAHIE-WILSON M.N., AHLQUIST J.A. : Hyperprolactinemia due to macroprolactins : Some progress but still a problem. Clin. Endocr., 2003, 58 : 683-685.

18. GARG R.K., KHAISHGI A., DANDONA P. : Is management with Sildenafil changing clinical practice ? Lancet, 1999, 353 : 375-376.

19. GUAY A.T., SABHARWAL P., VARMA S., MALARKEY W.B. : Delayed diagnosis of psychological erectile dysfunction because of the presence of macroprolactinemia. J. Clin. Endocr. Metab., 1996, $81: 2512-2514$.

20. HAAKE P., EXTON M.S., HAVERKAMP J. et al. : Absence of orgasm-induced prolactin secretion in a healthy multi-orgasmic male subject. int. J. Impot. Res., 2002, 14 : 133-135.

21. ISHIKAWA H., KANEKO S., OHASHI M., NAKAGAWA K., HATA M. : Retrograde ejaculation accompanying hyperprolactinemia. Arch. Androl., 1993, 30 : 153-155.

22. JOHNSON A.R., JAROW J.P. : Is routine endocrine testing of impotent men necessary ? J. Urol., 1992, $147: 1542-1543$.

23. JOHRI A.M., HEATON J.P.W., MORALES A. : Severe erectile dysfunction is a marker for hyperprolactinemia. Int. J. Impot. Res., 2001, 13 : 176-182.

24. KROPMAN R.F., VERDIJK R.M., LYCKLAMA A., NIJEHOLT
A.A.B., ROELFSEMA F. : Routine endocrine screening in impotence : significance and cost-effectiveness. Int. J. Impot. Res., 1991, $3: 87-94$.

25. LEMAIRE C., LEMAIRE A., DEWAILLY D., FOSSATI P. : Hyperprolactinemia with an excess of high molecular weight prolactin in men. Int. J. Impot. Res., 1994, 6 Suppt $1: 79$.

26. LEONARD M.P., NICKEL C.J., MORALES A. : Hyperprolactinemia and impotence: Why, when, and how to investigate? J. Urol., 1989, $142:$ 992-995.

27. LOBO R.A., KLETZKY O.A. : Normalization of androgen and sex-hormone-binding globulin levels after treatment of hyperprolactinemia. J. Clin. Endocr. Metab., 1982, 56 : 562-566.

28. MAATMAN T.J., MONTAGUE D.K. : Routine endocrine screening in impotence. Urology, 1986, $27: 499-502$.

29. MIYAI K., ICHIHARA K., KONDO K., MORI S. : Asymptomatic hyperprolactinemia and prolactinoma in the general population - Mass screening by paired assays of serum prolactin. Clin. Endocrinol., 1986, $25: 549-554$.

30. MIYAKE A., IKEGAMI M., CHEN C.F. et al. : Mass screening for hyperprolactinemia and prolactinoma in men. J. Endocrinol. Invest., 1988, $11:$ 373-384.

31. MOUNIER C., TROUILLAS J., CLAUSTRAT B., DUTHEL R., ESTOUR B. : Macroprolactinaemia associated with prolactin adenoma. Hum. Reprod., 2003, 18 : 853-857.

32. PASSOS V.Q., SOUZA J.J.S., MUSOLINO N.R.C., BRONSTEIN M.D. : Long-term follow-up of prolactinomas : Normoprolactinemia after bromocriptine withdrawal. J. Clin. Endocrinol. Metab., 2002, $87:$ 3578-3582.

33. PRIOR J.C., COX T.A., FAIRHOLM D., KOSTASHUK E., NUGENT R. : Testosterone-related exacerbation of a prolactin-producing macroadenoma : Possible role for estrogen. $\mathrm{J}$. Clin. Endocrinol. Metab., 1987, 64 : 391-394.

34. REHMAN J., CHRIST G., ALYSKEWYCZ M., KERR E., MELMAN A. : Experimental hyperprolactinemia in a rat-model : alteration in centrally mediated neuroerectile mechanisms. Int. J. Impot. Res., 2000, 12 : 23-32.

35. RHODEN E.L., ESTRADA C., LEVINE L., MORGENTALER A. : The value of pituitary magnetic resonance imaging in men with hypogonadism. J. Urol., 2003, $170: 795-798$.

36. SCHWARTZ M.F., BAUMAN J.E., MASTERS W.H. : Hyperprolactinemia and sexual disorders in men. Biol. Psychiat, 1982, $17: 861-876$.

37. SELMANOFF M. : Tyrosine hydroxylase and POMC mRNA in the arcuate region are increased by castration and hyperprolactinemia. Mol. Brain Res., 1991, $10: 277-281$.

38. SINHA Y.N. : Structural variants of prolactin : occurrence and physiological significance. Endocr. Rev., 1995, 16 : 354-369.

39. VALLETTE-KASIC S., MORANGE-RAMOS I., SELIM A. et al.: Macroprolactinemia revisited : a study on 106 patients. J.Clin. Endocrinol. Metab., 2002, 87 : 581-588.

40. VERMEULEN A., ANDO S., VERDONCK L. : Prolactinomas. Testosterone-binding globulin and androgen metabolism. J. Clin. Endocr. Metab., 1982, 54 : 409-413.

41. WOLFSBERGER S., CZECH T., VIERHAPPER H., BENAVENTE R., KNOSP E. : Microprolactinomas in males treated by transsphenoidal surgery. Acta Neurochir., 2003, 145 : 935941.

Manuscrit reçu: juin 2005 ; accepté juin 2005. 


\section{ABSTRACT}

Update on hyperprolactinemia and sexual function in men

Jacques BUVAT, Gilbert BOU-JAOUDE

Erectile dysfunction (ED), generally associated with reduced sexual desire and sometimes with orgasmic or ejaculatory dysfunction, is the major presenting symptom of hyperprolactinemia (HPRL) in men, a condition which should not be missed since many cases are due to pituitary tumors, likely to result in serious complications. It is generally believed that the mechanism of prolactin (PRL)-induced sexual dysfunction is a decrease in testosterone secretion. In fact, serum testosterone is normal in many hyperprolactinemic males and testosterone-independent mechanisms are also involved, probably mainly involving cerebral neurotransmitter systems. Systematic determinations of serum PRL have found very low prevalences of marked HPRL (> $35 \mathrm{ng} / \mathrm{ml})$ in ED patients $(0.76 \%$ in a compilation of more than 3,200 patients) and pituitary adenoma $(0.4 \%)$. In addition, the association of HPRL with ED may have been coincidental in some of these cases, since $10 \%$ of HPRLs diagnosed by the usual immunological assays are due to macroprolactins, which are biologically inactive or minimally active variants of PRL. Specific identification of PRL requires PRL chromatography which is only available in some specialized laboratories. No consensus has yet been reached concerning screening for HPRL in ED. Systematic determination of serum PRL may be justified, as HPRL is a serious but reversible disease, while there is presently no reliable clinical, psychometric or hormonal criteria (including serum testosterone level) allowing to restrict its determination to certain categories of ED patients without a risk of missing certain cases of HPRL. In the case of consistent HPRL, looking for hypothalamic or pituitary tumor is mandatory. Dopamine-agonist therapy is the first-line treatment for PRL-induced sexual dysfunction. Sexual counselling may be necessary for some patients.

Key words: hyperprolactinemia, erectile dysfunction, inhibited sexual desire, anorgasmia, retarded ejaculation, review 